\title{
EL OVILLEJO EN LA POESÍA DRAMÁTICA DE CALDERÓN
}

\author{
FAUSTA AnTONUCCI \\ Università Roma Tre \\ fausta.antonucci@uniroma3.it
}

El estudio de la polimetría calderoniana se puede abordar desde una pluralidad de perspectivas: desde la cronológica de Hilborn (1938), que examina la evolución de los usos métricos del dramaturgo para avanzar hipótesis sobre la datación de las obras sin fechar; a la funcional inaugurada por Marín (1982), que bucea en los usos dramáticos de determinadas estrofas; a la estructural que propuso Williamsen (1978), invitando a considerar las correspondencias que el uso de la misma estrofa establece entre escenas diferentes de la misma obra, y que luego erigió en sistema Vitse (1998) inquiriendo en la relación entre cambios de metro y cambios escénicos, considerados los primeros como factores estructurantes primarios de la obra teatral áurea. Pero me atrevo a decir, pidiendo venia por lo obvio y aparentemente simplista de la afirmación, que el primer paso y obligado es el del reconocimiento correcto de las formas métricas utilizadas por Calderón, a veces tergiversado en las ediciones, inclusive las más solventes (donde la sinopsis métrica puede faltar o presentar errores), y hasta en la clásica monografía de Hilborn (1938), a día de hoy todavía el único trabajo de conjunto dedicado a la polimetría calderoniana ${ }^{1}$.

En estas páginas me propongo estudiar el caso del ovillejo, composición que en todos los manuales de métrica española se vincula a Cervantes, quien al parecer lo inauguró: hay tres en el capítulo 27 de la primera parte del Quijote, donde los canta Cardenio, para asombro y delicia del cura y del barbero; y cuatro en $L a$ ilustre fregona, producto de la pluma de Tomás de Avendaño o Tomás Pedro, que el ventero lee a su mujer tras haberlos descubierto en el libro de la cuenta de la cebada. Si algún autor más del siglo XVII se menciona en dichos manuales en relación con este tipo de estrofa, es a sor Juana (Navarro Tomás, 1956: 272; Jauralde

1 Como ejemplo del descuido que ha afectado a algunos aspectos de la polimetría calderoniana, solo recordaré el caso de la copla real, cuya presencia en el teatro de Calderón ha sido detectada solo muy recientemente (Antonucci, 2019).

Edad de Oro, XL (2021), pp. 581-601, ISSN: 0212-0429 - ISSNe: 2605-3314

DOI: https://doi.org/10.15366/edadoro2021.40.026 
Pou, 2020: 428). En realidad, y antes de sor Juana, fue Calderón quien dio bastante cabida en sus obras teatrales al ovillejo: Alatorre (1990) rastrea esta estrofa en seis autos sacramentales, haciendo hincapié en sus peculiaridades y añadiendo en una nota final que también se encuentra en Eco y Narciso. Sánchez (1957) ya había señalado uno de los ovillejos que reseña Alatorre, el de Tu prójimo como a ti, así como el que figura en La fiera, el rayo y la piedra, que había traído a colación Dámaso Alonso (1951: 49 y ss.) como ejemplo de técnica diseminativo-recolectiva; y sugería que podía tratarse de una reminiscencia cervantina del dramaturgo, si no de un homenaje velado.

Al parecer, ningún otro dramaturgo antes de Calderón utilizó los ovillejos en sus obras; sin duda no lo hizo Lope, si nos atenemos a los datos que nos proporciona el clásico estudio de Morley y Bruerton (1940) sobre la versificación en la dilatada producción teatral del Fénix. Después de Calderón, al contrario, y además de sor Juana, señala Alatorre (1990: 636) algunos ejemplos novedosos de Agustín de Salazar y Torres. En cuanto a Calderón, además de los títulos señalados por Sánchez y Alatorre, he podido rastrear cuatro más que incluyen ovillejos (el corpus examinado coincide con el que se consigna y reseña a día de hoy en la base de datos Calderón Digital): el primero en orden cronológico es Las tres justicias en una, tragedia que ha sido datada entre 1630 y 1637; los demás, tras un importante intervalo temporal, son Darlo todo y no dar nada, El golfo de las sirenas y Fieras afemina amor, comedias de tema mitológico o de historia antigua compuestas para representaciones palaciegas entre 1651 y 1669. En las páginas que siguen voy a comentar los rasgos formales de estos ovillejos, en busca de sus peculiaridades; incidiré, sobre todo, en la relación que mantienen con las estrofas que los preceden y siguen, y en las diversidades estructurales que manifiestan, debidas tanto a su relación con la acción dramática, como al género en el que se enmarcan las piezas que los incluyen.

El ovillejo calderoniano más sencillo y corto es el que se encuentra en la segunda jornada de La fiera, el rayo y la piedra (1651). Aquí, en una breve secuencia que interrumpe la acción de los protagonistas humanos, Cupido se jacta de sus triunfos con su hermano Anteros, en un monólogo en silvas que se remata con este ovillejo, mitad cantado por la música, mitad recitado por Cupido (Calderón, 1989)²:

$\begin{array}{ll}\text { CuPIDO } & \text { Céfiro ¿en quién dicha espera? } \\ \text { MúsICA } & \text { En una fiera. } \\ \text { CuPIDO } & \text { ¿Y quién a Ifis da desmayo? } \\ \text { MúsICA } & \text { Un bello rayo. } \\ \text { CuPIDO } & \text { ¿En quién Pigmaleón no medra? } \\ \text { MúsICA } & \text { En una piedra. }\end{array}$

2 La edición, muy solvente, de Aurora Egido, de la que cito, carece de sinopsis métrica. 
CuPIDo Ninguno llegue a ser yedra

del laurel que ama, porque hoy

lloren todos, que yo soy

la fiera, el rayo y la piedra

(vv. 2.060-2.069).

La definición que del «ovillejo» nos proporciona el Diccionario de métrica española de Domínguez Caparrós (1999: s.v.) es la siguiente: 'Estrofa de diez versos dispuestos de la siguiente forma: tres pareados de octosílabo y quebrado; una redondilla octosílaba que sigue la rima del último pareado y cuyo último verso se forma con la unión de los tres quebrados. La rima es consonante'. La definición regulariza, obviamente, las singularidades que pueden observarse en los distintos textos y que el mismo Caparrós (2002: 148) observa ya en los modelos cervantinos. Vemos aquí, por ejemplo, que los versos cortos no son quebrados en sentido propio, es decir, tetrasílabos, sino pentasílabos (aunque cabe la posibilidad de una compensación por sinalefa con el verso anterior); y que el último verso reproduce solo una parte de cada verso corto. Pero la estructura de los tres pareados presenta el mismo esquema de pregunta (octosílabo) / respuesta (verso corto) que caracteriza los ovillejos fundacionales del Quijote y que es una de las características de esta composición, aunque algunas veces, como veremos, puede tergiversarse.

Mucho más articulados y complejos son los ovillejos que aparecen en la tercera jornada de Las tres justicias en $u_{n} a^{3}$. Se trata de una escena en la que Elvira, la criada de Violante, le pregunta a esta por su estado de ánimo, a raíz de la riña que han tenido don Lope padre e hijo, que aboca a don Lope hijo a la prisión y al castigo del rey, por lo que Violante, enamorada del joven, teme por su vida. En cuanto termina la secuencia en ovillejos, Elvira revela a su señora que Lope está preso en el cuarto de don Mendo, padre de Violante, por lo que esta decide en el acto que ayudará a su enamorado a fugarse. El diálogo en ovillejos entre señora y criada representa un momento de remanso en la acción, que se vuelve a poner en marcha inmediatamente después, precipitándose hacia la catástrofe final. Violante no llegará nunca a liberar a don Lope, porque el rey se le adelanta ejecutando personalmente en el joven la condena a muerte a la que lo ha sentenciado por haber afrentado públicamente a su padre. La secuencia está formada por cuatro

3 Curiosamente, Hilborn no reconoce estos ovillejos como composición con fisonomía propia, y los describe como «a unique type of metre, made up of rhymed couplets with alternating octosyllabics and verses of four or five syllables», algo que «certainly does not resemble in versification anything else in the comedias of Calderón», por lo que duda de la autoría calderoniana de la tercera jornada (1938: 40). Este desconocimiento es bien extraño, puesto que, como hemos visto, los ovillejos aparecen en cinco comedias más, que Hilborn examina bajo el aspecto métrico: en estos casos, sin embargo, no se merecen ningún comentario y terminan en el cajón de sastre de las formas métricas «Miscellaneous» bajo el mismo rótulo de «irregular» que marca el ovillejo de Las tres justicias en una. 
ovillejos, más tres redondillas que pueden considerarse como parte integrante de la misma secuencia métrica, porque resumen la situación discursiva (Violante ya no quiere contestar a las preguntas de Elvira) y porque la redondilla final reúne los últimos versos de los cuatro ovillejos anteriores; un recurso este que ya Alatorre (1990: 653-654) había observado en el auto El pintor de su deshonra llamándolo «super-recolección». He aquí la secuencia:

\begin{tabular}{|c|c|}
\hline ELVIRA & ¿De qué nace tu dolor? \\
\hline ViOLANTE & De un temor. \\
\hline ELVIRA & ¿Y el temor, señora, injusto? \\
\hline Violante & De un disgusto. \\
\hline ELVIRA & ¿Qué es, en fin, tu desconsuelo? \\
\hline \multirow[t]{2}{*}{ Violante } & Un recelo. \\
\hline & $\begin{array}{l}\text { Porque hoy ha dispuesto el cielo } \\
\text { que, a una tristeza rendida, } \\
\text { puedan quitarme la vida } \\
\text { temor, disgusto y recelo. }\end{array}$ \\
\hline ELVIRA & ¿Quién embaraza tu dicha? \\
\hline Violante & Mi desdicha. \\
\hline ELVIRA & Pues ¿quién causa su rigor? \\
\hline Violante & Mi amor. \\
\hline ELVIRA & Dime lo que te importuna. \\
\hline \multirow[t]{2}{*}{ Violante } & Mi fortuna. \\
\hline & $\begin{array}{l}\text { Y así, sin piedad alguna, } \\
\text { no hallo alivio en mi pasión, } \\
\text { porque mis contrarios son } \\
\text { desdicha, amor y fortuna. }\end{array}$ \\
\hline ELVIRA & ¿Quién alienta tu querella? \\
\hline ViOLANTE & Mi estrella. \\
\hline ELVIRA & Véncela con tu arrebol. \\
\hline Violante & Es mi estrella todo el sol. \\
\hline ELVIRA & Su luz eclipsa importuna. \\
\hline Violante & $\begin{array}{l}\text { Está menguante mi luna, } \\
\text { con que esperanza ninguna } \\
\text { me ha quedado; pues ya vi } \\
\text { conjurados contra mí } \\
\text { la estrella, el sol y la luna. }\end{array}$ \\
\hline ElviRA & ¿Qué te obliga a mal tan fuerte? \\
\hline Violante & Ver mi muerte. \\
\hline ELVIRA & Pues ¿quién tu muerte ha causado? \\
\hline Violante & ¿Quién? El hado. \\
\hline ELVIRA & Pierde, señora, el recelo. \\
\hline ViOLANTE & Es contra el cielo; \\
\hline
\end{tabular}




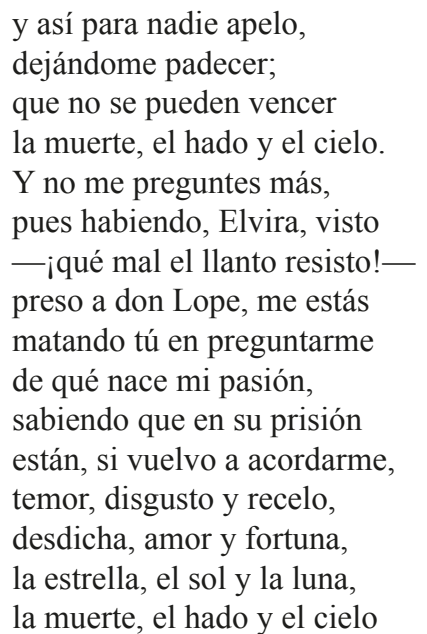

(Benabu, 1991: vv. 2.305-2.356) .

Observamos que los ovillejos tercero y cuarto no se conforman del todo con el típico esquema pregunta (octosílabo) / respuesta (quebrado), pues algunas parejas de versos (vv. 2.327-2.330, vv. 2.339-2.340) presentan un esquema exhortación / respuesta y están formadas por dos octosílabos. Muy interesante el rasgo metapoético del «si vuelvo a acordarme» (v. 2.352), con el que Violante parece hacer memoria de los cuatro versos conclusivos de los ovillejos anteriores, como si de un alarde de recitación se tratara.

Otros ovillejos interesantísimos, porque muestran claramente la disposición experimental e innovadora de Calderón para con estas «composiciones de ingenio», como las llama Navarro Tomás (1956: 271), son los que se encuentran en la tercera jornada de Eco y Narciso (1661). En este caso, la estructura del ovillejo, con algunas modificaciones, se combina con la del eco, como es lógico dado el tema de la pieza. Recordemos además, con Alatorre (1990: 662-663), que en el siglo XVII el nombre que se daba a la composición que nos ocupa era precisamente el de «eco», aunque no se tratara casi nunca de un verdadero «eco»; mientras el nombre de «ovillejos» indicaba los endecasílabos con rimalmezzo o, más corrientemente, la silva de pareados ${ }^{5}$.

Nos encontramos en el último cuadro de la obra, en el que culmina la tragedia de los dos protagonistas, propiciada, en la versión ideada por Calderón (que se aleja bastante de las fuentes clásicas), por la madre de Narciso, Liríope, que quiere protegerlo de un oráculo nefasto y acabará al contrario acelerando su muerte. La pérdida

4 Modernizo la grafía con respecto a la edición de Benabu.

5 De hecho, al estudiar la evolución de la silva de pareados en el siglo XVII, Alatorre llama esta forma métrica «ovillejos», aun aclarando que este rótulo no se generalizó en la época porque «competía con otras acepciones» (1988: 27). 
de la capacidad de habla autónoma en Eco se debe a que ha pisado un veneno poderosísimo preparado por Liríope que quiere alejarla de su hijo, pero al mismo tiempo, también al dolor y a los celos que le provoca ver a Narciso enamorado de su propia imagen reflejada en el agua de una fuente; la escena se merecería una cita completa por su eficacia dramática, ya que Eco no solo pierde la palabra, sino que de improviso pierde también la memoria de lo que está diciendo, primero de forma parcial, luego definitiva. El trastorno psíquico de la ninfa se traduce, pues, en una progresiva incompetencia que no es solamente lingüística sino también argumental. Todo esto se dramatiza en una larga secuencia de romance á-a que acoge las repeticiones de Eco como parte de los octosílabos del romance. Baste un ejemplo:

$\begin{array}{ll}\text { NARCISO } & \begin{array}{l}\text { ¿Qué preguntas, si me hablas? } \\ \text { Yo soy Narciso. }\end{array} \\ \text { ECO } & \text { Narciso. } \\ \text { NARCISO } & \begin{array}{l}\text { Sí. ¿Qué te espantas? } \\ \text { ECO }\end{array} \\ \text { ¿Espantas? } \\ \text { NARCISO } & \begin{array}{l}\text { Pues ¿no he de espantarme yo } \\ \text { al ver en ti tal mudanza? }\end{array} \\ & \text { ¿Qué ibas diciendo? } \\ \text { ECO } & \text { ¿Diciendo? } \\ \text { NARCISO } & \text { Sí, no calles nada. } \\ \text { ECO } & \text { Nada }\end{array}$

(Calderón, 2010: 210).

El mecanismo básico del ovillejo empieza a apuntar en la cuarteta cantada que se engasta más adelante en la misma secuencia en romance á-a ${ }^{6}$ :

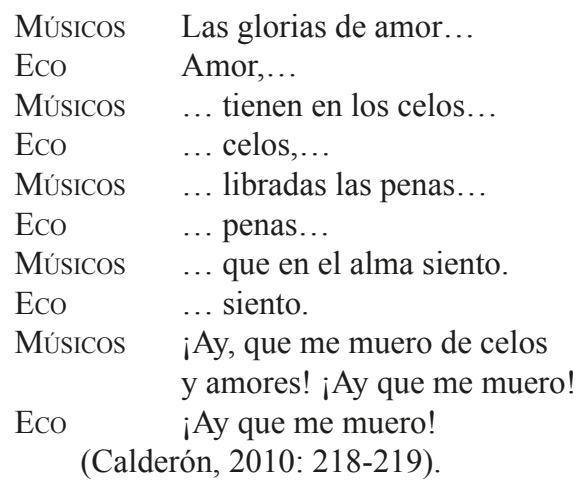

\footnotetext{
6 He modificado algo la puntuación con respecto a la edición de la que cito, aquí y en las citas sucesivas extraídas de Eco y Narciso.
} 
Las repeticiones de Eco transforman el romancillo hexasílabo é-o en romance é-o (asonancia esta que, como observa Kroll [2019], es precisamente la de Eco). Sin embargo, Narciso se da cuenta de que no se trata de meras repeticiones, sino que, como sucede en el ovillejo, cobran un sentido autónomo si se consideran conjuntamente:

\begin{tabular}{|c|c|}
\hline NARCISO & $\begin{array}{l}\text { De suerte que repetidos } \\
\text { de esos versos los finales, } \\
\text { alguien lamenta sus males, } \\
\text { diciendo en otros sentidos: } \\
\text { «Amor, celos, penas siento. } \\
\text { ¡Ay que me muero!» } \\
\text { (Calderón, 2010: 218-21s }\end{array}$ \\
\hline
\end{tabular}

Finalmente, el ovillejo aparece en su modalidad más reconocible en el momento culminante del drama: los avisos de Eco a Narciso, que se va dando cuenta gracias a ellos de su muerte ya próxima ${ }^{7}$ :

$\begin{array}{ll}\text { NARCISO } & \text { Pues, Eco, oye, aunque tú mueras... } \\ \text { ECO } & \ldots \text { mueras... } \\ \text { NARCISO } & \ldots \text { celosa, yo enamorado... } \\ \text { ECO } & \ldots \text { enamorado... } \\ \text { NARCiSO } & \ldots \text { no me he acordar de ti. } \\ \text { ECO } & \ldots \text { de ti. } \\ \text { NARCISO } & \text { Mas, iay, cielos!, que si aquí } \\ & \text { junto las voces que oí, } \\ & \text { ioh, madre!, y las consideras, } \\ & \text { en tres voces dijo: «Mueras } \\ & \text { enamorado de ti», } \\ & \text { y temo que la oiga el cielo... } \\ \text { ECO } & \ldots \text { el cielo... } \\ \text { NARCISO } & \ldots \text { pues es fuerza que me dé... } \\ \text { ECO } & \ldots \text { me dé... } \\ \text { NARCISO } & \ldots \text { de mí mismo a mí venganza. } \\ \text { ECO } & \ldots \text { venganza. } \\ \text { NARCISO } & \text { Y más agora que alcanza } \\ & \text { a ver mi desconfianza }\end{array}$

7 Cabe subrayar que, desde la perspectiva del editor de textos, el reconocimiento del ovillejo es imprescindible para evitar casos como el de la edición «urgente» de Eco y Narciso que aparece en la BVMC <http://www.cervantesvirtual.com/obra-visor/eco-y-narciso--0/html/ff3ec8e8-82b111df-acc7-002185ce6064_7.html\#I_0_>, que edita este pasaje como si octosílabos y quebrados formaran parte de un mismo verso, lo que tergiversa tanto la forma del ovillejo como la correcta numeración de los versos. 


\begin{tabular}{|c|c|}
\hline & $\begin{array}{l}\text { que, lo último repitiendo } \\
\text { de mi acento, está diciendo: } \\
\text { «El cielo me dé venganza». } \\
\text { Esta imposible hermosura... }\end{array}$ \\
\hline EcO & $\ldots$ hermosura... \\
\hline Narciso & ... y aquella hermosura y voz... \\
\hline Eco & $\ldots y$ voz... \\
\hline NARCISO & ... a un mismo tiempo me han muerto... \\
\hline EcO & $\ldots$ muerto... \\
\hline NARCISO & $\begin{array}{l}\text {... pues tan claramente advierto } \\
\text { que, oráculo del desierto, } \\
\text { cuando a mis penas compite, } \\
\text { Eco conmigo repite: } \\
\text { «Hermosura y voz me han muerto». } \\
\text { ¡Ay de mí, infeliz, que muero! }\end{array}$ \\
\hline EcO & ... muero... \\
\hline NARCISO & Y mi misma sombra amando,... \\
\hline Eco & $\ldots$ amando... \\
\hline NARCISO & $\ldots$ una voz aborreciendo,.. \\
\hline EcO & $\ldots$ aborreciendo... \\
\hline NARCISO & $\begin{array}{l}\text {... con que se está averiguando } \\
\text { que el hado va ejecutando } \\
\text { sus amenazas. Huir quiero } \\
\text { de mí mismo, pues ya «muero } \\
\text { aborreciendo y amando» } \\
\text { (Calderón, 2010: 221-222). }\end{array}$ \\
\hline
\end{tabular}

En esta secuencia el ovillejo es, de hecho, un eco, pues los quebrados, en lugar de ser respuestas a los interrogantes planteados en los octosílabos, como en el ovillejo típico, son repeticiones literales de la última porción del octosílabo. Por tanto, los tres pareados que caracterizan la primera parte de cada ovillejo presentan una rima idéntica. Además, se rematan con una quintilla aabba en lugar de la redondilla típica, con algunas licencias en la recapitulación final de los quebrados, que en dos casos se extiende sobre dos versos, en un caso invierte el orden de las palabras, en otro añade un verbo auxiliar que la repetición no contemplaba.

Nos queda por comentar un recurso sobre el que volveremos más adelante. Al marcharse Narciso, tras pronunciar los últimos versos citados, los ovillejos dejan paso otra vez al romance é-o en una réplica de Liríope que se remata con los dos versos finales de los dos ovillejos últimos, ligeramente modificados: 


\section{LiRíope ¡Oh, qué en vano los mortales quieren entender al cielo! Todos los medios que puse para estorbar los empeños hoy de su destino han sido facilitarlos más presto, pues la voz de Eco le aflige $y$, por venir della huyendo, muerte le da su hermosura, con que ya cumplido veo que hermosura y voz le matan, amando y aborreciendo}

(Calderón, 2010: 221-222; énfasis mío).

Se trata de un recurso recolectivo parecido al que hemos observado en Las tres justicias en una, en las redondillas que constituyen una coda de los ovillejos, y que vamos a observar nuevamente en Darlo todo y no dar nada.

La segunda jornada de Darlo todo y no dar nada (1651) se abre con un larguísimo cuadro ambientado en el bosque, espacio dramático en el que se suceden diversas peripecias. Campaspe, que iba a cazar, salva la vida a Alejandro, cuyo caballo se había desbocado; cuando el rey se retira a descansar en la quinta cercana donde viven las hijas de Darío, sus prisioneras, Campaspe, cansada por el esfuerzo y afligida por el recuerdo de Apeles, que le ha salvado la vida en una ocasión anterior y al que no ha vuelto a ver, se duerme; en ello sale al tablado Apeles, que se desespera por saber algo de Campaspe y se pregunta qué habrá sido de ella. Las preguntas de Apeles en su monólogo parecen tener respuesta en las palabras inconexas que Campaspe, también preocupada por la suerte de Apeles, pronuncia en sueños ${ }^{8}$ :

$\begin{array}{ll}\text { APELES } & {[\ldots] \text { ¿Murió en faltándola yo? }} \\ & \text { Habla entre sueños Campaspe } \\ \text { CAMPASPE } & \text { No. } \\ \text { APELES } & \text { ¿Tuvo, cuando ausente estuve... } \\ \text { CAMPASPE } & \text { Tuve... } \\ \text { APELES } & \ldots \text { quien venciese en su disculpa? } \\ \text { CAMPASPE } & \ldots \text { la culpa. } \\ \text { APELES } & \text { ¿Qué eco a mi voz respondió? } \\ \text { CAMPASPE } & \text { Yo. } \\ \text { APELES } & \text { ¡Cielos! ¿Si es verdad o no } \\ & \text { que el aire me ha respondido? } \\ & \text { Pues ha sonado en mi oído... } \\ \text { Los dos } & \text {... «no tuve la culpa yo». }\end{array}$

8 Aquí también modifico en algunos lugares la puntuación. 


$\begin{array}{ll}\text { APELES } & \text { ¿Si oí bien o mal habrá quien... } \\ \text { CAMPASPE } & \text { Bien... } \\ \text { APELES } & \ldots . \text { me diga, y si verdad fue... } \\ \text { CAMPASPE } & \ldots \text { que... } \\ \text { APELES } & \ldots \text { que en mi desdicha fue dicha? } \\ \text { CAMPASPE } & \ldots \text { la desdicha... } \\ \text { APELES } & \text { ¿Tuvo amparo cuando anduve? } \\ \text { CAMPASPE } & \begin{array}{l}\text { Tuve. } \\ \text { APELES }\end{array} \\ & \begin{array}{l}\text { Otra vez fuerza es que hube } \\ \text { de dudar, si es que colijo }\end{array} \\ & \text { que el eco otra vez me dijo... } \\ \text { Los DOS } & \text {... «bien que la desdicha tuve». } \\ \text { APELES } & \begin{array}{l}\text { Mas no, ilusión es ligera, } \\ \text { que el eco no habló en lo hueco; }\end{array} \\ & \begin{array}{l}\text { pues no me dijera el eco } \\ \text { lo que yo no le dijera }\end{array} \\ & \text { (Calderón, 1956: } 1.043 \text { ). }\end{array}$

El pasaje es sumamente interesante por diversas razones. En primer lugar, los dos ovillejos que presenta se componen cada uno de cuatro pareados de octosílabo y verso corto, en lugar de los tres canónicos. Estos pareados obedecen al esquema pregunta / respuesta que caracteriza el ovillejo, pero de forma algo diferente con respecto a los ejemplos de La fiera, el rayo y la piedra y Las tres justicias en una. De hecho, la voz de Campaspe parece contestar directamente a las preguntas de Apeles cuando estas se contienen en un solo verso (es el caso de «¿Murió en faltándola yo? / No», «¿Qué eco a mi voz respondió? / Yo», «¿Tuvo amparo cuando anduve? / Tuve»); pero en los demás casos, su voz no tiene una relación discursiva tan directa con las dudas que se plantea Apeles, limitándose a aprovechar la consonancia para enunciar breves porciones que luego formarán una oración completa en el último verso de la redondilla final. En la última réplica de Apeles el personaje apunta a la relación entre el eco y el ovillejo, desdiciéndose de lo que ha dado por supuesto poco antes, es decir, que la voz que le contesta sea un eco. De hecho, si en el primer ovillejo los versos cortos son eco de los octosílabos en tres casos (estuve / tuve, disculpa / culpa, respondió / yo), ya en el segundo ovillejo no hay tal eco, y Apeles entiende por tanto que quien le contesta es una voz humana. Por ello, se pone a buscar «el dueño» de esta voz $\mathrm{y}$, al poco, da con Campaspe dormida y la despierta. La joven, asustada porque cree ver a un fantasma, reacciona así:

$\begin{array}{ll}\text { Campaspe } & \text { ¡Ay de mí! } \\ & \text { ¿Qué miro! } \\ \text { APeles } & \quad \text { ¿Qué mal anduve! }\end{array}$




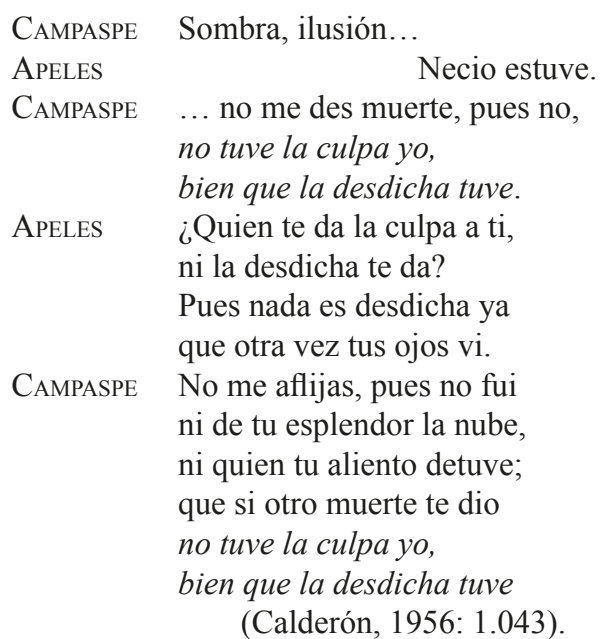

En este caso, la «super-recolección», para seguir utilizando la definición de Alatorre, se encuentra bastante después de que hayan terminado los ovillejos, cerrando la tercera y la cuarta de las nueve décimas que siguen a los ovillejos y concluyen la escena. De hecho, es como si los ovillejos se proyectaran sobre estas décimas en más de un aspecto. El típico entramado de preguntas largas (octosílabas) y respuestas breves (trisílabas, tetrasílabas o a lo sumo pentasílabas) que caracteriza el ovillejo, se traslada a algunos versos octosílabos partidos de la sexta y la novena décima:

\begin{tabular}{|c|c|}
\hline CAMPASPE & ¿Luego no eres sombra? \\
\hline APELES & $\begin{array}{ll}\text { No. } & \text { No }\end{array}$ \\
\hline Campaspe & ¿Luego estás con vida? \\
\hline APELES & Sí. \\
\hline CAMPASPE & ¿No te mataron? \\
\hline APELES & No fui \\
\hline & $\tan$ dichoso. \\
\hline CAMPaSPE & ¿Dicha fuera? \\
\hline APELES & $\begin{array}{l}{[\ldots]} \\
\text { ¿Será posible lo sea } \\
\text { el volver a verte? }\end{array}$ \\
\hline CAMPASPE & Sí. \\
\hline APELES & ¿Dónde he de buscarte? \\
\hline CAmPaspe & Aquí. \\
\hline APELES & ¿Vendrás? \\
\hline Campaspe & Hablad, alma, vos. \\
\hline APELES & ¿Qué dices? \\
\hline CAMPASPE & $\begin{array}{c}\text { Que sí. [...] } \\
\text { (Calderón, 1956: 1.043-1.044). }\end{array}$ \\
\hline
\end{tabular}


Vemos aquí, pero también lo observamos en Las tres justicias en una y en Eco y Narciso, que la secuencia en ovillejos no puede aislarse de las secuencias en otros metros que la preceden o siguen inmediatamente. Y si volvemos a considerar el caso de La fiera, el rayo y la piedra, vemos que allí también el ovillejo forma una unidad inseparable con el pasaje en silva de pareados que lo precede y que introduce los temas que el ovillejo trata luego de forma más incisiva.

Observamos el mismo mecanismo en la secuencia formada por ovillejos y redondillas que caracteriza métricamente el momento en que el bajel con Ulises y sus compañeros entra en el golfo de las sirenas, en la égloga piscatoria homónima (1657), y se acerca a las criaturas cuyo canto atrae irremediablemente a los marineros:

\begin{tabular}{|c|c|}
\hline ULISES & ¿Quién canta en el mar tan bien,... \\
\hline SIRENA 1 & ... quién? \\
\hline ULISES & ¿Cuando otra vez me destierra... \\
\hline SIRENA 2 & $\ldots$ de tierra... \\
\hline ULISES & ... de que yo escapar pretendo... \\
\hline SIRENA 3 & ... huyendo... \\
\hline ULISES & ... porque a mi honor le conviene? \\
\hline SIRENA 4 & Viene. \\
\hline DANTE & Misterio el eco contiene. \\
\hline ANTEO & $\begin{array}{l}\text { No es eco. ¿No ves veloces } \\
\text { sirenas decir a voces }\end{array}$ \\
\hline TODAS & ¿Quién de tierra huyendo viene...? \\
\hline Ulises & ¿De quién pretendo yo huir? \\
\hline SIRENA 1 & De oír. \\
\hline ULISES & ¿Qué más intento vencer? \\
\hline SIRENA 2 & Y ver. \\
\hline ULISES & Pues, ¿quién tiene por disgusto... \\
\hline SIRENA 3 & $\ldots$ gusto... \\
\hline Ulises & ... que yo a mí me quiera dar? \\
\hline SIRENA 4 & Pesar. \\
\hline ANTEO & $\begin{array}{l}\text { Sentido tray singular } \\
\text { el canto que nos persigue. }\end{array}$ \\
\hline DANTE & Sí, pues dice que se sigue \\
\hline TODAS & de oir y ver, gusto y pesar. \\
\hline Ulises & Pues si me juzgué muriendo... \\
\hline SIRENA 1 & ... viendo, \\
\hline ULISES & ... un peligro a otro añadiendo... \\
\hline SIRENA 2 & ... oyendo, \\
\hline ULISES & $\ldots$ durar mi dolor cruel... \\
\hline SIRENA 3 & ... en él... \\
\hline ULISES & ¿no era morir y no amar? \\
\hline
\end{tabular}




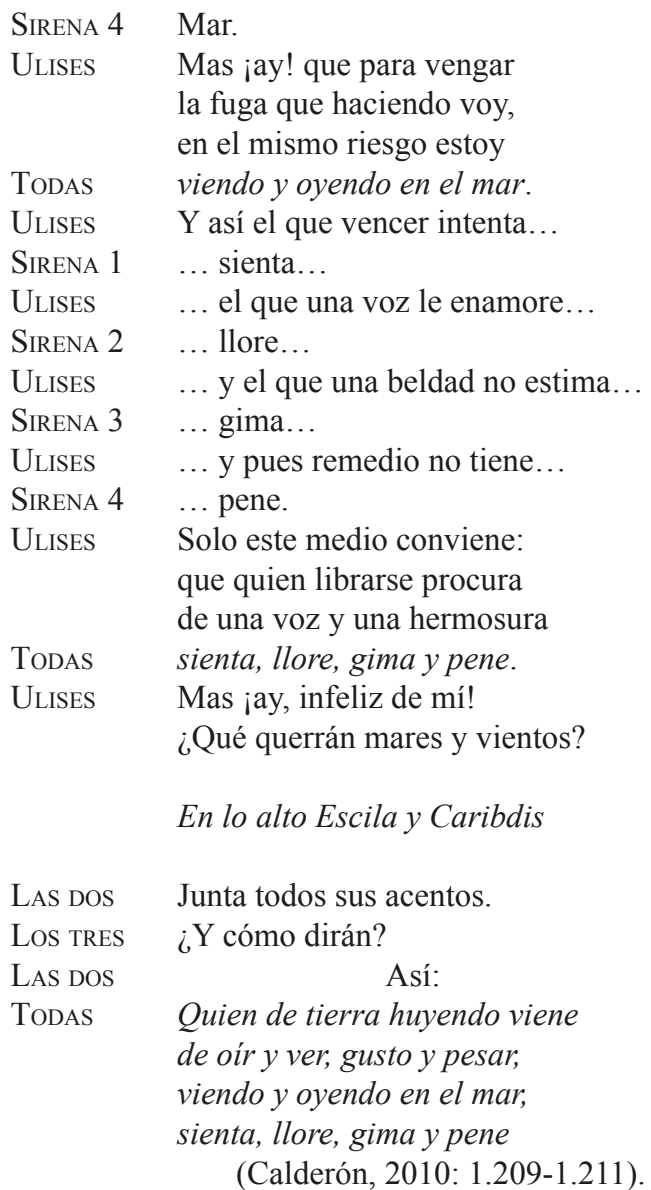

Como en Darlo todo y no dar nada, aquí los ovillejos están formados por cuatro pareados en lugar de los clásicos tres. Los octosílabos que cierran cada uno de los cuatro ovillejos se repiten, en la «super-recolección» que ya podemos considerar característica de Calderón, en la segunda de las cuatro redondillas que siguen a los ovillejos, antes de dar paso a una secuencia en romance. También encontramos la alusión metapoética al nombre de la composición, en la breve discusión al final del primer ovillejo entre Anteo y Dante acerca de si se trata o no de un eco: de hecho, solo el segundo y el cuarto quebrado se configuran como eco del octosílabo anterior.

La relación entre octosílabos y quebrados se nos aparece aquí más compleja que en Darlo todo y no dar nada, donde se observaba cierta desvinculación del verso corto con respecto al octosílabo precedente. Al contrario, en 
El golfo de las sirenas los quebrados, cuando no contestan directamente a preguntas expresadas en los octosílabos (como en los dos primeros pareados del segundo ovillejo), contribuyen a la formación del sentido de la oración que se pone en marcha en los octosílabos. Dicho en otras palabras, la sucesión de octosílabos no significaría gran cosa, incluso a veces no tendría significado, sin la colaboración de los quebrados, como se ve si se considera con atención el primer ovillejo, en el que solo el último quebrado no completa semánticamente el octosílabo anterior, funcionando como mero eco de este. El esquema pregunta / respuesta que caracteriza el ovillejo en su forma más típica parece haber dejado paso a otro esquema, en el que el quebrado tiende a precisar, desarrollar o completar la oración iniciada en el octosílabo: un ejemplo clarísimo lo tenemos en el cuarto ovillejo. Evidentemente un esquema como este solo puede funcionar en la atmósfera mágica que caracteriza las comedias mitológicas, y que justifica el que las sirenas sepan no solo contestar las preguntas, sino también completar las frases apenas esbozadas de Ulises con pleno conocimiento de lo que le ha sucedido. Se trata de una modalidad discursiva que no sería compatible con una acción dramática verosímil, como la de Las tres justicias en una y Darlo todo y no dar nada; de hecho, en esta última, la compatibilidad semántica entre los versos pronunciados por Apeles y los pronunciados por Campaspe es parcial y está limitada a la correspondencia entre preguntas y respuestas. Correspondencia que por otra parte solo se da por casualidad, propiciada por la coincidencia en las preocupaciones de los dos jóvenes y justificada por el hecho de que Campaspe está soñando, siendo el sueño un momento privilegiado de visión divinatoria, pero no necesariamente vinculado con la magia.

Nos confirma en esta hipótesis el análisis de los ovillejos que se encuentran en el cuadro inicial de la tercera jornada de Fieras afemina amor (1669), ambientado en el jardín de las Hespérides. Al final de una larga secuencia en romance é-o, Yole, desesperada porque teme que Hércules mate a su amado Anteo, como de hecho sucederá, se pregunta qué hará si este sucumbe. Es el comienzo de una serie de ovillejos, en los que, a las preguntas de Yole, contestan cantando Venus y Cupido (a los que ella no ve), exhortándola a fingir amor por el héroe que así se verá castigado por su rechazo de este sentimiento. Por comodidad, y para facilitar el comentario, pues se trata de un pasaje bastante difícil, doy también la numeración de los versos y escribo en cursiva los versos finales de cada ovillejo, así como la «super-recolección» final, que aquí se repite dos veces y que ocupa dos de las cuatro redondillas que rematan, como en Las tres justicias en una, el pasaje en ovillejos: 
Yole

ENUS

Yole

CuPIDO

Yole

CuPIDO

Yole

ELLA Y LOS DOS

Yole

CUPIDO

Yole

VENUS

Yole

CuPIDO

Yole

ELLA Y LOS DOS

Yole

VENUS

Yole

CuPIDO

Yole

VENUS

Yole

ELLA Y LOS DOS

Yole

CupIDO

Yole

Venus

Yole

CuPIDO

Yole

ELLA Y LOS DOS

Yole
[...] ¿Qué haré si él llega a morir?

Venus y Cupido, cantando a sus lados, sin verlos.

Fingir...

¿Qué puede fingir mi estrago?

... halago...

Y ¿qué será ese favor?

... traidor.

Eco, ya que a mi dolor

de oráculo eres trasunto,

si él muere, ¿qué haré?, pregunto.

Fingir halago traidor.

¿Más alivio a mis sospechas...

... que con flechas...

... en fingir halagos das?

... más...

¿Que serán no consideras...

... severas?

Mal con voces lisonjeras

persuades a mis rencores

vengarse antes con favores

que con flechas más severas.

Dime, anuncio más cruel,...

... que él...,

¿qué obra halago que se aplica?

... domestica...

¿Quién dirá que de él lo esperas?

... las fieras.

¿Cómo es posible que quieras,

dudando si vence o no

Hércules, que escuche yo

que él domestica las fieras?

Y pues son vanas quimeras...

... fieras...

... el presumir que su ruina...

... afemina...

... dime si hay medio mejor.

... Amor.

Permite que mi temor

crédito a tu voz no dé,

pues nada consuela oír que

fieras afemina Amor.

$\mathrm{Si}$ ya, viendo mi dolor, 


$\begin{array}{ll} & \text { junto todo no te obligas } \\ & \text { a que de una vez me digas } \\ & \text { qué medio me está mejor. } \\ \text { Los Dos } & \text { Fingir halago traidor; } \\ & \text { que con flechas más severas } \\ & \text { que él domestica las fieras, } \\ & \text { fieras afemina Amor. } \\ & \text { Pues si el favor } \\ \text { que por consejo me das } & 3.255 \\ \text { Yole fingir, desde hoy verás, } \\ \text { viéndome contra un furor, } \\ \text { fingir halago traidor, } \\ \text { que con flechas más severas } \\ \text { que él domestica las fieras, } \\ \text { fieras afemina Amor } \\ \quad \text { (vv. 3.214-3.269). }\end{array}$

En esta cita, tanto como en la anterior de El golfo de las sirenas, he cambiado en muchas ocasiones la puntuación propuesta por la edición de la que cito (Calderón, 1984) para resaltar mejor la relación discursiva entre octosílabos y quebrados: un reto indudablemente para el editor. Como ya observaba en El golfo de las sirenas, aquí también en algunos casos (sin ir más lejos, en el primer ovillejo) los versos cantados por Cupido y Venus funcionan como respuestas a las preguntas de Yole, según el esquema clásico de esta composición. Pero ya a partir del segundo ovillejo, la relación entre preguntas y respuestas, versos octosílabos y versos cortos, se hace más complicada: los versos que cantan Venus y Cupido, de hecho, completan los que pronuncia Yole, a veces contestando a sus dudas, otras veces integrando lo dicho por ella. Un caso patente es el de los vv. 3.224-3.229, que no se entienden si no se acepta que los vv. 3.224-3.226 forman en conjunto una pregunta, cuya respuesta se encuentra en el v. 3.227, y que a su vez los vv. 3.228-3.229 forman otra pregunta. Otro ejemplo se observa en el último ovillejo, donde los versos cantados por Venus y Cupido, que forman al final el título de la obra (Fieras afemina amor), aparecen, en los dos primeros pareados, como parte integrante y necesaria de los versos de Yole, y solo en el último pareado vuelven a adquirir el carácter de respuesta a un interrogante. No hace falta insistir en que aquí también estamos en el marco de una comedia mitológica, donde lo mágico y sobrenatural se manifiestan en la presencia de dioses y diosas, que en este caso le insuflan a Yole una solución a su dilema que es también su propia venganza contra Hércules. Se observa asimismo que, una vez más, a la voz que contesta a sus preguntas Yole la llama «Eco», aunque en realidad en esta secuencia ninguno de los quebrados es eco efectivo del octosílabo que lo precede. 
Por supuesto, cualquier descubrimiento ulterior de ovillejos en la producción dramática calderoniana podrá servir para matizar o incluso reformular las breves conclusiones que propongo a continuación, que por tanto han de entenderse como provisionales, aunque recuerdo que están basadas en un análisis de más de la mitad de los títulos de autoría segura o probable que se le adjudican al dramaturgo. Lo primero que me parece oportuno subrayar es que los ovillejos no funcionan como forma métrica autónoma, al estilo de lo que sucede por ejemplo con las décimas o las coplas reales, para limitarme a citar otras estrofas de arte menor caracterizadas por una mayor complejidad con respecto a la redondilla o la quintilla. Los ovillejos se proyectan siempre, como he tratado de mostrar, en la secuencia estrófica sucesiva, generalmente en redondillas, que acoge esa «super-recolección» que observara Alatorre en los autos sacramentales y que, si excluimos el caso de La fiera, el rayo y la piedra, aparece en todas las demás obras con ovillejos que hemos examinado, aunque con modalidades diferentes. Y si no hay tal proyección, como en el caso apenas mencionado, sí existe una vinculación estrechísima con la forma estrófica precedente, de la que los ovillejos representan una suerte de remate.

En la producción calderoniana, hasta donde llegan hoy mis conocimientos, la presencia del ovillejo se concentra en un periodo de menos de veinte años (16511669), quedando muy alejada de esta franja cronológica la primera pieza en la que se utiliza, Las tres justicias en una, que ha sido fechada tentativamente por su editor entre 1630 y 1637 (Benabu, 1991: 3-6). Quizás, si no se encontraran más títulos con ovillejos entre estas fechas y 1651, podría reconsiderarse la hipótesis de datación que ha sido avanzada, recordando que en mayo de 1644 el autor de comedias Pedro Ascanio se compromete a representarla en Valencia describiéndola como una «comedia nueva, nunca representada en Valencia» (Cruickshank, 2011: $245)^{9}$, y que ya Alexander Parker (1962: 229) había propuesto para su datación la horquilla 1635-1640.

Desde el punto de vista funcional, tan importante o más que el formal o el cronológico, me parece poder decir que el ovillejo lo reserva Calderón para momentos álgidos de la trama dramática: aun cuando en cada pieza la calidad de estos momentos cambia, en relación con el desarrollo propio, sí podemos decir que, en los títulos considerados, nunca utiliza Calderón los ovillejos en la primera jornada o al comienzo de la segunda.

En cuanto a la manera como se construyen los ovillejos, a la «forma» de la relación entre octosílabos y quebrados, y a su calidad discursiva, hemos podido ver en qué medida Calderón se va alejando del esquema típico del que se considera el espécimen de esta composición, el primer ovillejo de Cardenio en el capítulo 27

\footnotetext{
9 No creo que hubiese podido definirla «comedia nueva» si se hubiera remontado su composición a los primeros años treinta.
} 
de la primera parte del Quijote. Este alejamiento — que ya Alatorre había observado en los ovillejos de sus autos sacramentales - es el resultado del constante afán de experimentación que caracteriza la polimetría calderoniana, y que se intensifica a partir de los años cincuenta del siglo (Antonucci, en prensa). Sin duda, la experimentación métrica calderoniana recibe un impulso importante a raíz de su dedicación cada vez más exclusiva, a partir de estas fechas, a la composición de obras de gran aparato destinadas a representaciones palaciegas, en las que la presencia de la música era parte integrante y fundamental del espectáculo. Aunque no podamos contrastar los textos de El golfo de las sirenas y Fieras afemina amor con la música que se compuso para las partes cantadas, que no se ha conservado (Stein, 1993), es plausible que las modificaciones que hemos observado en los ovillejos que se engastan en dichas obras obedezcan en parte a exigencias musicales. Por otra parte, creo que dichas modificaciones también se deben, como he apuntado arriba, a la atmósfera mágica que impregna la acción y que influye en el carácter discursivo propio del ovillejo, alejándolo en parte del conocido esquema pregunta / respuesta y haciendo que los versos cortos, pronunciados por seres sobrenaturales, se integren muchas veces de forma novedosa, casi oracular, en los versos pronunciados por el personaje humano (Ulises y Yole, en los casos que hemos analizado). 
BIBLIOGRAFÍA

Alatorre, Antonio (1988). «Quevedo: de la silva al ovillejo». En Luisa López Grigera y Augustin Redondo (eds.), Homenaje a Eugenio Asensio. Madrid: Gredos, pp. 19-31. Alatorre, Antonio (1990). «Perduración del ovillejo cervantino». Nueva Revista de Filología Hispánica, 38: 2, pp. 643-674.

Alonso, Dámaso (1951). «Tácticas de los conjuntos semejantes en la expresión literaria». En Dámaso Alonso y Carlos Bousoño, Seis calas en la expresión literaria española (Prosa - poesía - teatro). Madrid: Gredos, pp. 45-74.

Antonucci, Fausta (2019). «La copla real en el teatro de Calderón». Arte Nuevo. Revista de Estudios Áureos, 6, pp. 1-20<https://www.artenuevorevista.com/index.php/artenuevo/article/view/85/279> [Consulta: 16/4/2021].

Antonucci, Fausta (en prensa). «Propuestas para una reconsideración de conjunto de la polimetría calderoniana». En Siglo de Oro: nuevas perspectivas. Actas del XII Congreso de la Asoción Internacional del Siglo de Oro. Kassel: Reichenberger.

Benabu, Isaac (1991). On the boards and in the press. Calderón's "Las tres justicias en una». Kassel: Reichenberger.

Calderón de la Barca, Pedro (1956). Obras completas. II. Dramas. Ángel Valbuena Briones (ed.). Madrid: Aguilar.

Calderón de la Barca, Pedro (1984). Fieras afemina Amor. Edward M. Wilson (ed.). Kassel: Reichenberger.

Calderón de la Barca, Pedro (1989). La fiera, el rayo y la piedra. Aurora Egido (ed.). Madrid: Cátedra.

Calderón de la Barca, Pedro (2010). Cuarta parte de comedias. Sebastian Neumeister (ed.). Madrid: Fundación José Antonio de Castro.

Calderón Digital. Base de datos, argumentos y motivos del teatro de Calderón. Fausta Antonucci (dir.) <http://calderondigital.tespasiglodeoro.it/> [Consulta: 28/03/2021].

Cruickshank, Don W. (2011). Calderón de la Barca. Su carrera secular. José Luis Gil Aristu (trad.). Madrid: Gredos.

Domínguez CAParrós, José (1999). Diccionario de métrica española. Madrid: Alianza editorial.

Domínguez Caparrós, José (2002). Métrica de Cervantes. Alcalá de Henares: Centro de Estudios Cervantinos.

Hilborn, Harry Warren (1938). A Chronology of the Plays of D. Pedro Calderón de la Barca. Toronto: University of Toronto Press.

Jauralde Pou, Pablo (2020). Métrica española. Madrid: Cátedra.

Kroll, Simon (2019). «Espejos sonoros en Eco y Narciso: un análisis cuantitativo y poético de la asonancia en Calderón». Bulletin of the Comediantes, 71: 1-2, pp. 155-169.

Marín, Diego (1982). «Función dramática de la versificación en el teatro de Calderón». Segismundo, 35-36, pp. 95-113.

Morley, S. Griswold y Courtney Bruerton (1968). Cronología de las comedias de Lope de Vega. María Rosa Cartes (trad.). Madrid: Gredos.

NAVARro Tomás, Tomás (1956). Métrica española. Reseña histórica y descriptiva. Madrid: Ediciones Guadarrama. 
PARKer, Alexander A. (1962). «Towards a Definition of Calderonian Tragedy». Bulletin of Hispanic Studies, 39, pp. 222-237.

SÁnchez, Alberto (1957). «Reminiscencias cervantinas en el teatro de Calderón». Anales cervantinos, 6, pp. 262-270.

Stern, Louise K. (1993). Songs of Mortals, Dialogues of the Gods. Music and Theatre in Seventeenth-Century. Spain. Oxford: Clarendon Press.

VITSE, Marc (1998). «Polimetría y estructuras dramáticas en la comedia de corral del siglo XVII: el ejemplo de El Burlador de Sevilla». En Ysla Campbell (ed.), El escritor y la escena VI. Estudios sobre teatro español y novohispano de los Siglos de Oro. Ciudad Juárez: Universidad Autónoma de Ciudad Juárez, pp. 45-63.

WiLliamsen, Vern G. (1978). «The structural function of polymetry in the Spanish Comedia». En Alba V. Ebersole (ed.), Perspectivas de la Comedia. Chapel Hill: Estudios de Hispanófila, pp. 33-47.

Recibido: 21/04/2021

Aceptado: 24/05/2021 


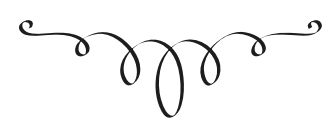

\section{El oviLlejo EN LA POESÍA DRAMÁTICA DE CALDERÓN}

Resumen: Se estudia la presencia del ovillejo en seis obras dramáticas de Calderón (Las tres justicias en una, La fiera, el rayo y la piedra, Darlo todo y no dar nada, El golfo de las sirenas, Eco y Narciso, Fieras afemina Amor). Se analizan sus características métricas y dialógicas, subrayando cómo va evolucionando con el transcurso de los años y en razón del género dramático de la pieza en que se engloba.

PALABRAS ClaVE: Calderón, métrica, ovillejo.

\section{The OVILLEJo IN CALDERÓN'S DRAMATIC POETRY}

ABSTRACT: In this paper we study the presence of the ovillejo in six dramatic works by Calderón (Las tres justicias en una, La fiera, el rayo y la piedra, Darlo todo y no dar nada, El golfo de las sirenas, Eco y Narciso, Fieras afemina Amor). Its metric and dialogic characteristics are analyzed, underlining how it evolves over the years and due to the dramatic genre of the piece in which it is included.

KEYwords: Calderón, metrics, ovillejo. 